\title{
Estudo Prospectivo Sobre Microencapsulamento de Óleos Vegetais no Brasil e no Mundo: uma análise até 2013
}

\author{
Prospective Study on Vegetable Oils Microencapsulation in Brazil and \\ Worldwide: an analysis by 2013
}

\author{
Camila Duarte Ferreira Ribeiro ${ }^{1}$ \\ Luciane Santos Sousa ${ }^{1}$ \\ Ísis Maria Pereira Borges ${ }^{1}$ \\ Itaciara Larroza Nunes ${ }^{1,2}$ \\ ${ }^{1}$ Universidade Federal da Bahia, Salvador, BA, Brasil \\ ${ }^{2}$ Universidade Federal de Santa Catarina, Florianópolis, Brasil
}

\begin{abstract}
Resumo
A modificação tecnológica dos óleos vegetais tem sido cada vez maior em decorrência da importância nutricional e econômica desses produtos. Portanto, o presente estudo prospectivo teve como objetivo avaliar o panorama mundial relativo ao microencapsulamento de óleos vegetais, correlacionando-o com os documentos depositados de patentes. Para tanto, realizou-se uma pesquisa das patentes nas bases de dados Espacenet ${ }^{\circledR}$ e INPI. Pode-se observar que houve um crescimento exponencial com pico em 2012 em relação ao depósito de patentes, sendo a China o país líder em número de patentes depositadas. Em relação à área de atuação, a indústria farmacêutica lidera, seguida da alimentícia. A técnica de microencapsulamento mais utilizada é a spray-drying, e os materiais de parede mais empregados são a maltodextrina e o amido. Conclui-se que há uma tendência ao crescimento de depósito de patentes referentes ao microencapsulamento de óleos. No Brasil, o número de patentes depositadas ainda é pequeno.
\end{abstract}

Palavras-chave: Micropartículas. Tecnologia. Inovação.

\begin{abstract}
The technological modification of vegetable oils has been increasing due to the nutritional and economic importance of these oils. Therefore, the present prospective study had as objective to evaluate the panorama regarding the microencapsulation of vegetal oils, correlating it with the deposited documents of patents. For this purpose, a patents search the in the databases Espacenet ${ }^{\circledR}$ and INPI was carried out. It can be observed that there was an exponential growth with peak in 2012 in relation to the filing of patents, with China being the leading country in number of patents deposited. In relation to the area of operation, the pharmaceutical industry leads, followed by food. The most commonly used microencapsulation technique is spray-drying and the most commonly used wall materials are maltodextrin and starch. It is concluded that there is a trend towards patent filing for oils microencapsulation. In Brazil, the number of patents deposited is still small.
\end{abstract}

Keywords: Microparticles. Technology. Innovation.

Área Tecnológica: Prospecção. Pesquisa. Inovação. 


\section{Introdução}

Os óleos têm um papel fundamental na alimentação humana, além de fornecerem calorias, eles agem como veículo para as vitaminas lipossolúveis, como A, D, E e K. Também são fontes de ácidos graxos essenciais como o linoleico, linolênico e araquidônico e contribuem para a palatabilidade dos alimentos. Os componentes mais expressivos dos óleos são os triacilgliceróis, e suas propriedades físicas dependem da estrutura e da distribuição dos ácidos graxos presentes (CASTRO et al., 2004).

Em 2019, a produção mundial de óleos girou em torno de 204 milhões de toneladas, sendo os dois principais o óleo de palma (72 milhões de toneladas) e o óleo de soja (56 milhões de toneladas). Os maiores produtores são a Indonésia (48 milhões de toneladas), a China (26 milhões de toneladas), a Malásia (21 milhões de toneladas), a União Europeia (18 milhões de toneladas), os Estados Unidos (12 milhões de toneladas), a Argentina (10 milhões de toneladas) e o Brasil (9 milhões de toneladas). O Brasil é o quinto maior produtor de óleos com 13 milhões de toneladas, com destaque para o óleo de palma, 540.000 toneladas (FAS 2020a; FAS, 2020b).

Cerca de $90 \%$ da produção de óleos são de origem vegetal, provenientes do processamento de sementes e destina-se ao consumo humano (FARIA, 2002; MENDONÇA; RAETANO; MENDONÇA, 2007). Na indústria, verifica-se um aumento na demanda de mercado em relação a óleos das mais diversas fontes naturais, destacando-se as aplicações em derivados alimentícios que cobrem a formulação de produtos, como bolos, biscoitos, pães, margarinas, produtos lácteos, uso na fritura, entre outras aplicações (CAR; HOOG, 2005; JEYARANI; YELLA REDDY, 2003).

Os $10 \%$ restantes da produção de óleos são destinados à produção de ração animal e utilizados em vários processos industriais, como matéria-prima na fabricação de fungicidas (MENDONÇA; RAETANO; MENDONÇA, 2007) sabões, detergentes, sabonetes, amaciantes biodegradáveis, cosméticos, medicamentos, entre outros (CAR; HOOG, 2005; JEYARANI; YELLA REDDY, 2003; BROCK et al., 2008; CONCEIÇÃO et al., 2005). O uso de óleos vegetais transesterificados, em detrimento dos óleos minerais originados de uma fração da destilação do petróleo, tem sido uma alternativa para a substituição de combustíveis derivados de petróleo, além de serem uma fonte de grande impacto econômico e social, uma vez que estes compõem o biodiesel (MENDONÇA; RAETANO; MENDONÇA, 2007; FROEHNER; LEITHOLD; LIMA JÚNIOR, 2007).

Devido à importância nutricional e econômica dos óleos comestíveis, nos últimos 15 anos houve um crescente interesse em processos tecnológicos que visam à modificação de características para adequar tais processos a uma determinada aplicação (CASTRO et al., 2004). Um exemplo prático é a obtenção do aumento da estabilidade oxidativa dos óleos por meio de microencapsulamento.

O microencapsulamento é uma técnica que tem sido utilizada para obtenção de óleos na forma de pó estável, dispersível em água e de fácil incorporação aos alimentos. Além disso, trata-se de uma técnica promissora para preservação de óleos contra a oxidação, uma vez que limita influências indesejáveis do ambiente, como oxigênio, luz, umidade, entre outros, aumentando, assim, a sua vida útil (AGHBASHLO et al., 2013; SHEN et al., 2010).

Além de bastante utilizada pela indústria alimentícia, a microencapsulação vem sendo estudada e empregada em outras áreas industriais, sobretudo no setor farmacêutico, o qual 
permitiu o desenvolvimento de fórmulas de liberação controlada de fármacos que apresentam a capacidade de liberar o agente ativo apenas no local ou órgão em que este deve agir. Outra área de intensa pesquisa é o setor de agrotóxicos, que busca diminuir a toxicidade e a contaminação ambiental com a criação de fórmulas de liberação controlada de defensivos agrícolas. A microencapsulação ainda encontra inúmeras aplicações nos setores de cosméticos, pigmentos, adesivos, encapsulação de células vivas, entre outros (SUAVE et al., 2006).

Essa tecnologia consiste em empacotar partículas em tamanhos que podem ir de 0,2 $\mu \mathrm{m}$ a $5.000 \mu \mathrm{m}$, como compostos de sabor, pigmentos, acidulantes, nutrientes, enzimas, conservantes, entre outros (AGHBASHLO et al., 2013), sendo o material encapsulado denominado de recheio, núcleo ou core, e o material que forma a cápsula, de encapsulante, cobertura, parede ou carreador (AZEREDO, 2005; PARIZE, 2009). A parede tem como principal função o aumento da vida útil do core, retardando a isomerização e a oxidação desses compostos, preservando, dessa forma, suas características. Além disso, outro importante objetivo do microencapsulamento é permitir que a liberação do encapsulado ocorra lentamente com o tempo, ou a partir da ocorrência de um certo evento. A liberação controlada ajuda a evitar a utilização desnecessária e a perda de compostos durante o processamento (AZEREDO, 2005).

Existem inúmeras técnicas de microencapsulamento, como coacervação, spray-cooling, extrusão, extrusão centrífuga, recobrimento em leito fluidizado, lipossomas e complexação por inclusão (GOUIN, 2004). No entanto, o spray-drying é uma das técnicas mais utilizadas na indústria de alimentos para microencapsular óleos devido ao baixo custo no processo e pela alta produtividade conferida (CARNEIRO et al., 2013; MOREIRA, 2007).

Os materiais de parede mais utilizados para encapsular óleos são as gomas, especialmente goma arábica ou acácia, maltodextrinas de diferentes equivalentes de dextroses (DE), amidos modificados, caseinato de sódio, proteína do leite, proteína isolada da soja e suas misturas (AGHBASHLO et al., 2013).

Na literatura predominam trabalhos sobre o encapsulamento de óleo de peixe (AGHBASHLO et al., 2013; BARRET et al., 2001; HEINZELMANN; FRANKE, 1999; JAFARI et al., 2008; KAGAMI et al., 2003; KEOGH et al., 2001; POLAVARAPU et al., 2011; WANG; TIAN; CHEN, 2011), sendo encontrados também alguns referentes ao encapsulamento de óleo de linhaça (CARNEIRO et al., 2013; GALLARDO et al., 2013; QUISPE-CONDORI; SALDAÑA; TEMELLI, 2011; TONON; GROSSO; HUBINGER, 2011). Além disso, há estudos que relacionam o encapsulamento de óleo de palma (DIAN; SUDIN; YUSOFF, 1996; FERREIRA et al., 2016a), azeite de oliva (CALVO et al., 2010), óleo de girassol (AHN et al., 2008; DOMIAN et al., 2014), óleo de cânfora (CHANG et al., 2006), entre outros.

No cenário da tecnologia relativa ao microencapsulamento, para manutenção de empresas numa situação economicamente viável em um ambiente de competitividade, faz-se necessário o fator inovação. Nesse contexto, o domínio sobre o fluxo de informações e o conhecimento das tendências tecnológicas futuras e a antevisão de possíveis rupturas no padrão de competição de uma dada indústria se convertem em uma poderosa ferramenta de competitividade e de racionalização dos esforços de capacitação das empresas. Para sistematizar as informações referentes às inovações tecnológicas que podem identificar as oportunidades e necessidades relevantes para a pesquisa no futuro, são realizados os trabalhos de prospecção (OLIVEIRA et al., 2012).

Ressalta-se que a busca se deu até 2013, pois, a partir do ano seguinte, foram publicados os primeiros trabalhos que envolveram o encapsulamento de óleo de palma bruto aqui no Brasil 
(FERREIRA et al., 2014; FERREIRA et al., 2016a) e esse mapeamento preliminar foi importante para a elaboração dos documentos de patentes que se sucederam em decorrência dessa busca (FERREIRA et al., 2016b; FERREIRA et al., 2016c). Diante do exposto e do grande interesse nacional e internacional, principalmente no ramo da indústria alimentícia, sobre os benefícios do microencapsulamento de óleos, o presente estudo prospectivo tem como objetivo avaliar o panorama mundial relativo ao tema em questão, correlacionando-o com os documentos de patentes depositados.

\section{Metodologia}

As patentes foram pesquisadas na base europeia Espacenet, visto que nessa base é possível encontrar patentes publicadas por mais de 90 países, e também na base dados do Instituto Nacional da Propriedade Industrial (INPI) para investigar as que foram depositadas aqui no Brasil.

Para o levantamento de dados na base europeia Espacenet, foram utilizadas palavras-chave associadas aos códigos da Classificação Internacional de Patentes (CIP), conforme demonstrado no Quadro 1. Esses códigos foram usados com o intuito de obter um maior número de resultados em relação aos documentos de patentes depositadas e possuem as seguintes descrições: B01J13 - Química coloidal, Fabricação de microcápsulas ou microbalões; A01N25 - Biocidas, repelentes ou atrativos de pestes ou reguladores do crescimento de plantas caracterizados por suas formas ou por seus ingredientes inativos ou por seus métodos de aplicação; A61K9 - Preparações medicinais caracterizadas por formas físicas especiais; B41M5 - Métodos de duplicação ou marcação.

As patentes que apresentaram os descritores que mais se associaram ao tema proposto, "Microencapsulamento de Óleos", em destaque no Quadro 1, foram selecionadas para o tratamento de dados. Foram utilizados os mesmos descritores para o levantamento de dados na base do INPI.

Quadro 1 - Busca de patentes por palavras-chave e agrupamento das palavras e códigos da IPC na base de dados europeia (Espacenet)

\begin{tabular}{|c|c|}
\hline TeRmos & PATENTES \\
\hline Microencapsulation & 1.285 \\
\hline Microencap* & 5.062 \\
\hline Microencap* and Oil* & $\mathbf{5 1 7}$ \\
\hline Microencap* and Oil & 429 \\
\hline Microencapsulation and Oil* & 175 \\
\hline Microencapsulation and Oil & 161 \\
\hline Microencap* and Oil* and B01J13 & 88 \\
\hline Microencap* and Oil* and A61K9 & 44 \\
\hline Microencap* and Oil* and B41M5 & 41 \\
\hline Microencap* and Oil* and A01N25 & 22 \\
\hline
\end{tabular}

Fonte: Elaborado pelos autores deste artigo (2017) 
Dos 517 documentos de patentes encontrados até 2013 na base de dados europeia, 354 estavam disponíveis e foram exportados para o programa CSVed 2.2.3 e, desse último, para o Microsoft Excel. A partir daí, foram criadas planilhas e gráficos referentes ao código de classificação internacional, distribuição de depósitos por país detentor da tecnologia, evolução anual das patentes, depósitos de patentes por empresas e inventores.

Cada planilha gerou um gráfico com representação dos 10 itens com maior incidência, com exceção do gráfico referente ao ano de publicação das patentes (de 1988 até 2013). Neste último, foram demonstrados apenas os 20 anos mais recentes em que houve depósito de patentes. Em relação à base de dados nacional, o INPI, foram encontrados 20 documentos de patentes, dos quais foram compiladas informações importantes, gerando gráficos como resultados. Dessa forma, foram analisadas no total 374 patentes (Espacenet + INPI).

\section{Resultados e Discussão}

Os resultados e a discussão foram apresentados de forma a trazer as características gerais e as mais específicas a respeito do objeto de estudo. Eles foram descritos nos tópicos seguintes.

\subsection{Evolução Anual das Patentes}

O Gráfico 1 apresenta a evolução anual de patentes depositadas referentes ao microencapsulamento de óleos no mundo e no Brasil. O primeiro registro ocorreu em 1964 por Alan Paul Osborne, na Grã-Bretanha e apresenta um método para aprimorar a colagem de borracha têxtil (OSBORNE, 1964).

No Brasil, a primeira patente identificada por essa busca foi requerida em 1988 por Papierfabrik August Koehler, da Alemanha, e trata-se de um método de microencapsulamento de óleos hidrofóbicos com partes reativas de sistemas de corantes (KOEHLER, 1988).

Gráfico 1 - Evolução mundial e brasileira do depósito de patentes entre os anos de 1988 e 2013

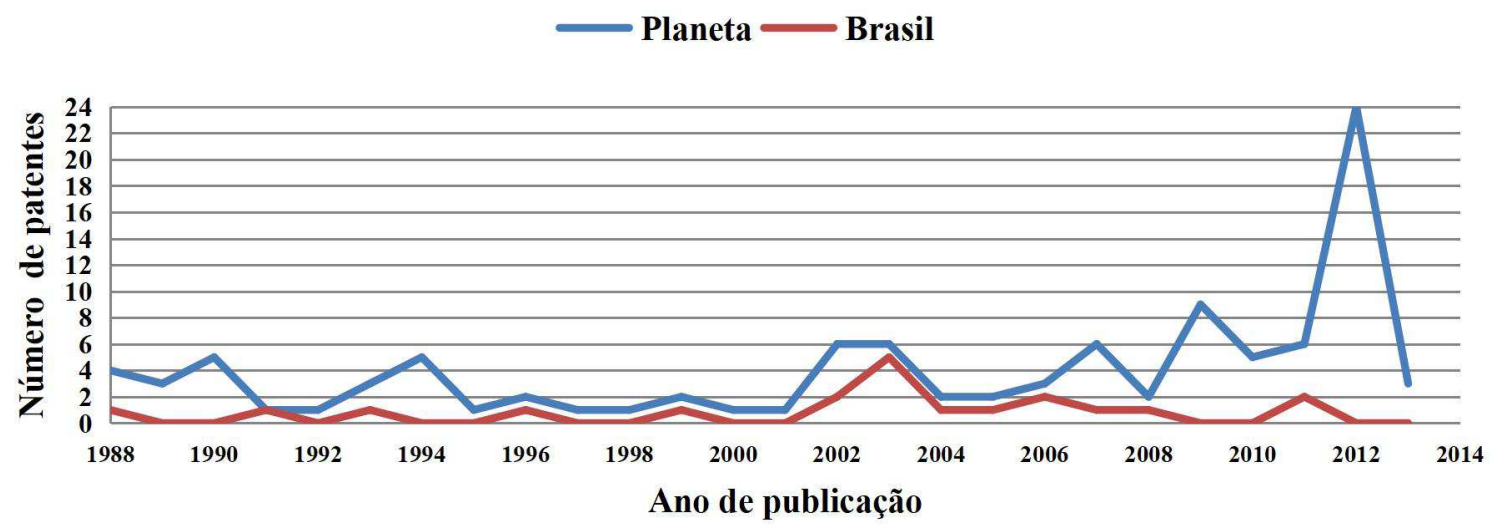

Fonte: Elaborado pelos autores deste artigo (2020)

Pode-se constatar que, de 1988 até 2013, houve um crescimento exponencial com pico em 2012 no que se refere ao depósito de patentes, tanto nos diversos países quanto no Brasil. O número de patentes genuinamente brasileiras só deve ser avaliado após 2002, quando o Brasil 
começou a se apropriar com mais continuidade dessa temática, sendo que o pico de 2003 e a queda de 2004 parecem indicar que ainda é necessário que a cultura de apropriação se torne mais regular, ou seja, não apenas mais difundida como também mais valorizada e exigida pelas agências de fomento brasileiras e pelo Estado Brasileiro.

Segundo Gouin (2004), em 2002, mais de 1.000 patentes envolveram processos de microencapsulação e suas aplicações, sendo que destas, 300 estavam associadas à microencapsulação de ingredientes de alimentos.

De maneira geral, a partir de 2000, houve um aumento discreto referente ao número de patentes sobre microencapsulamento de óleos, o que possivelmente corresponde à maturação da visão de desenvolvimento tecnológico, típica de uma tecnologia emergente e com potencial futuro. Ainda não foi atingido um patamar estável, evidenciando que a tecnologia é realmente emergente, tendo ainda alta potencialidade de desenvolvimento tecnológico (QUINTELLA et al., 2009).

\subsection{Classificação Internacional de Patentes (CIP)}

A Classificação Internacional de Patentes, conhecida pela sigla IPC - International Patent Classification - foi estabelecida pelo Acordo de Estrasburgo em 1971 e prevê um sistema hierárquico de símbolos para a classificação de Patentes de Invenção (PI) e de Modelo de Utilidade (MU), de acordo com as diferentes áreas tecnológicas a que pertencem. A IPC é adotada por mais de 100 países e coordenada pela Organização Mundial da Propriedade Intelectual (OMPI). O objetivo da busca e identificação da IPC nos documentos está relacionado com a facilidade de reconhecer a área de aplicação tecnológica desses documentos em nível internacional, independentemente da língua que o documento de patente foi escrito e depositado. Os códigos de classificação que mais apareceram nos documentos de patentes do mundo (Espacenet) selecionados estão inseridos na Seção A - Necessidades Humanas (Agricultura, Saúde; Divertimento e Alimentos; Tabaco) e na Seção B - Operações de processamento; Transporte (Separação: Mistura e Impressão) (Gráfico 2).

Gráfico 2 - Distribuição dos códigos da classificação internacional - Espacenet

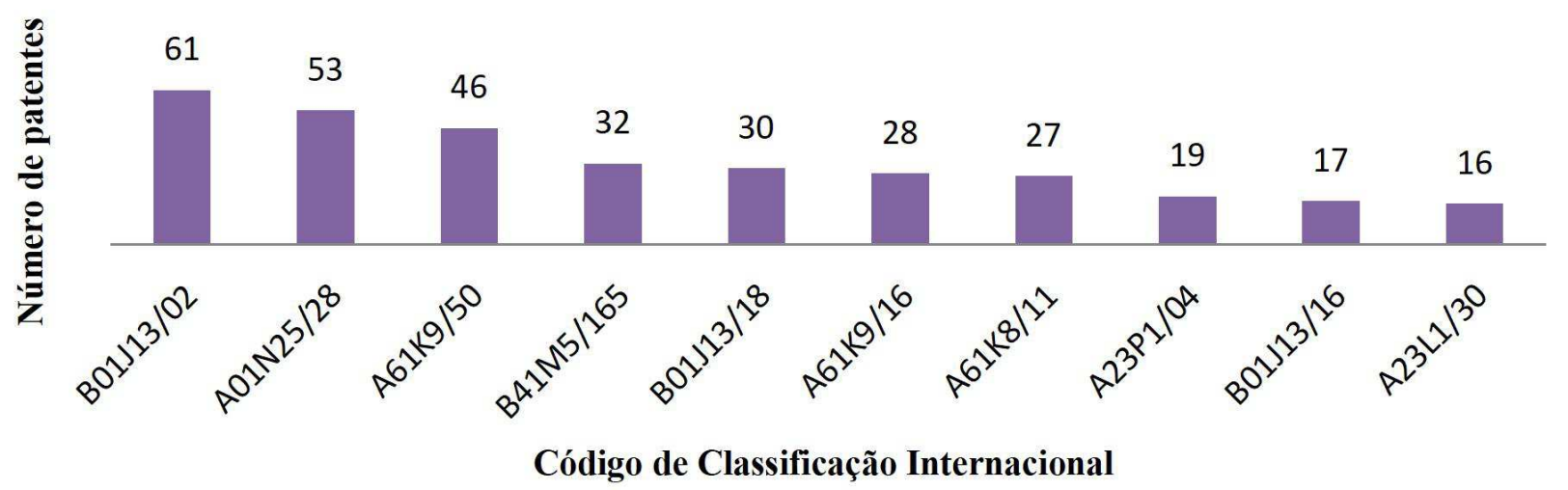

Fonte: Elaborado pelos autores deste artigo (2020) 
É possível afirmar que, entre as subdivisões no Espacenet, o maior número de patentes se refere ao código B01J13/02, referente à fabricação de microcápsulas ou de microbalões, com 61 registros. No INPI, os códigos de classificação que mais apareceram nos documentos de patentes selecionados também estão inseridos nas Seções A e B, respectivamente.

Comparando os códigos mais utilizados do mundo e do Brasil, este último tem mais patentes em A61K 36/48, A61K 7/46 e A01N 25/28, com duas patentes publicadas em cada um envolvendo, respectivamente, processo de microencapsulamento de composto volátil; absorção de óleos essenciais e extratos aromáticos em quitosana para ingestão; e microencapsulamento de composto agroquímico. Isso mostra que há uma diversidade nas apropriações dessa tecnologia no país. Esses resultados não eram esperados, visto que a principal utilização da tecnologia de sistemas de liberação controlada se concentra na área farmacêutica, de alimentos e de agrotóxicos (SUAVE et al., 2006).

\subsection{Perfil das Patentes}

O perfil das patentes apresenta de forma detalhada as características dos documentos de patentes encontrados, conforme se apresenta nos tópicos seguintes.

\subsubsection{Materiais Encapsulados}

Do volume total de documentos de patentes estudados, $26,27 \%$ e $10 \%$ referentes ao Espacenet e INPI, respectivamente (Gráfico 3A e Gráfico 3B), utilizou, de fato, óleos vegetais como encapsulados; 10,73\% e 20\% referentes ao Espacenet e INPI, respectivamente, utilizaram outros tipos de óleos (animal - óleo de peixe, fígado de ganso, foca; mineral - combustível; lubrificante); 35,02\% das patentes do Espacenet utilizaram outros encapsulados, como corante, bactéria, polímero, enquanto no INPI nenhuma patente utilizou outro encapsulado; $27,96 \%$ das patentes do Espacenet tratam sobre algum processo, $70 \%$ das patentes brasileiras tratam sobre esse assunto.

Diante do exposto, pode-se constatar que entre os 26,27\% (Gráfico 4) de patentes que utilizaram óleos vegetais no Espacenet, $6 \%$ foram de soja e gengibre, $4 \%$ de sândalo e canela, $3 \%$ de limão, cravo e cártamo, 33\% utilizaram outros óleos vegetais, como gérmen de trigo, linhaça, eucalipto, prímola, alecrim, cártamo, perila, erva-doce, cânfora, girassol, laranja doce, louro, azeite de oliva, gergelim, própolis, entre outros, e 38\% não especificaram o tipo de óleo vegetal. Nenhuma das patentes brasileiras que utilizaram óleo vegetal como encapsulado especificou o tipo. 
Gráfico 3 - Detalhamento das patentes encontradas no Espacenet (A) e no INPI (B)
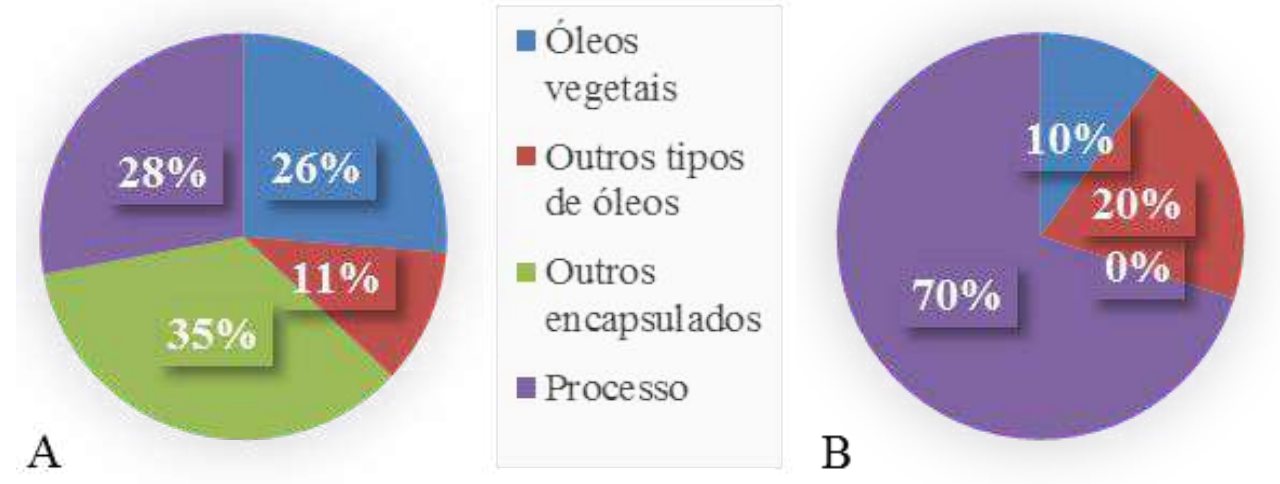

nóleos vegetais

- Outros tipos de óleos

Outros encapsulados

- Processo

Fonte: Elaborado pelos autores deste artigo (2020)

Gráfico 4 - Tipos de óleos vegetais encontrados nas patentes do Espacenet

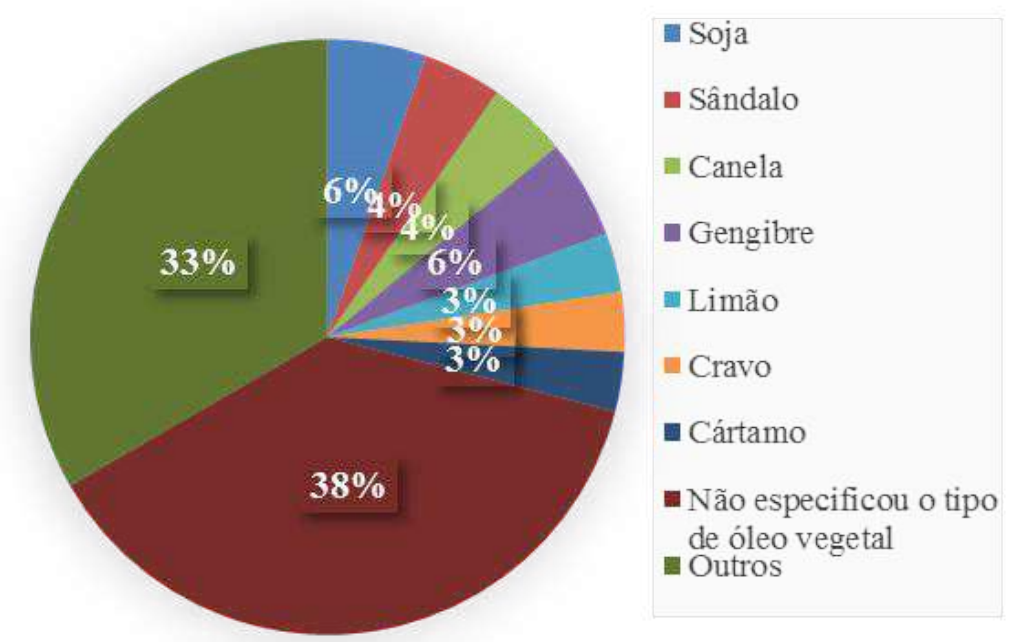

Fonte: Elaborado pelos autores deste artigo (2020)

\subsection{2 Área de Aplicação}

Em relação às patentes que, de fato, utilizaram óleos vegetais microencapsulados, a partir do estudo de Suave et al. (2006), foi possível determinar suas principais áreas de aplicação. O Gráfico 5 mostra a distribuição das principais áreas tecnológicas de aplicação do microencapsulamento de óleos vegetais, levando-se em consideração os documentos de patentes depositados até 2013. Pode-se notar que 15\% e 29\% das patentes do Espacenet e INPI, respectivamente, relacionam-se à agricultura (agrotóxicos, fertilizantes, pesticidas); 29\% e 17\% das patentes dessas bases de dados, respectivamente, relacionam-se à indústria de alimentos; $42 \%$ e $37 \%$ das patentes do Espacenet e INPI, respectivamente, relacionam-se à indústria farmacêutica (fármacos, fitoterápicos e cosméticos); 5\% e 17\% das patentes do Espacenet e INPI, respectivamente, relacionam-se a outras áreas de aplicação, como tratamento de água, agente de limpeza, tabaco, tubulação, tinta a jato, indústrias automobilística e têxtil, e $9 \%$ das patentes do Espacenet não especificaram a área de aplicação. 
Diante disso, é possível aferir que as principais pesquisas que envolvem o depósito de patentes de microencapsulamento de óleos vegetais, ou seja, a principal área de interesse está relacionada às propriedades farmacológicas dessa matriz. Tal resultado assemelha-se ao reportado por Suave et al. (2006).

Gráfico 5 - Áreas de aplicação das patentes encontradas no Espacenet (A) e no INPI (B)

A

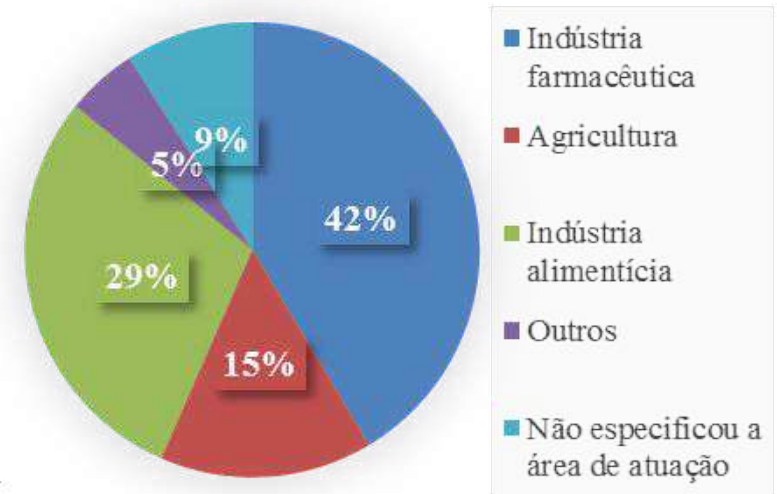

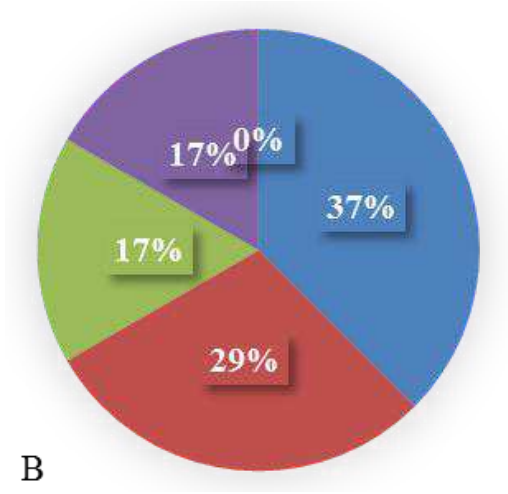

Indústria farmacêutica

Agricultura

- Indústria alimentícia

= Outros

Não especificou a área de atuação

Fonte: Elaborado pelos autores deste artigo (2020)

\subsubsection{Tecnologia Empregada}

Apesar de o método de coacervação ser o mais empregado para microencapsulação de partículas (SUAVE et al., 2006), segundo Carneiro et al. (2013), spray-drying é o processo amplamente utilizado para microencapsular óleos. No entanto, de acordo com o Gráfico 6, pode-se observar que, segundo o Espacenet, das patentes que envolveram o microencapsulamento de óleos vegetais, $67 \%$ não informaram a tecnologia empregada; $13 \%$ utilizaram spray-drying; $14 \%$ são de coacervação; $2 \%$ de liofilização e $4 \%$ empregaram outros métodos de microencapsulamento. Nenhuma das patentes brasileiras informava a tecnologia de microencapsulamento empregada, o que possivelmente pode ser justificado pelo fato de que boa parte delas envolve o processo de microencapsulamento, e o sigilo dessa informação acaba sendo importante.

Gráfico 6 - Tecnologia de microencapsulação empregada nas patentes do Espacenet
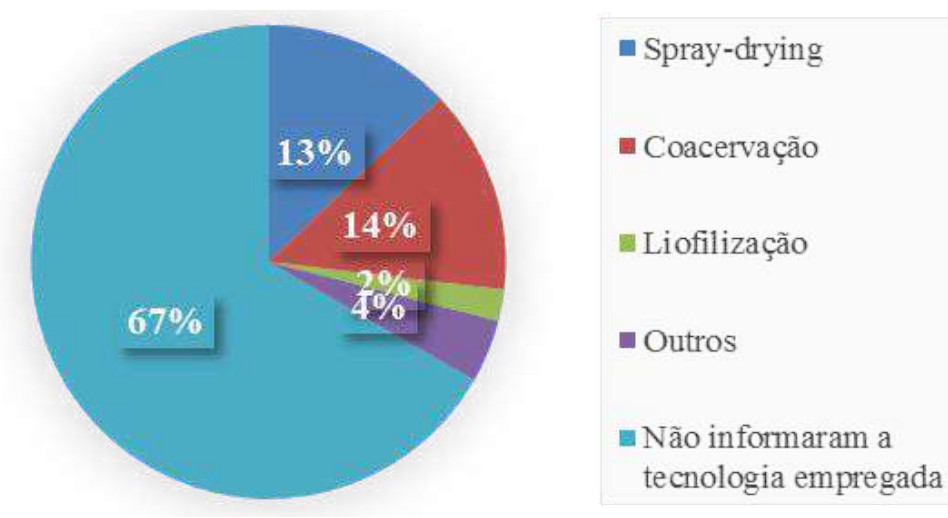

Fonte: Elaborado pelos autores deste artigo (2020) 


\subsubsection{Materiais de Parede}

Em relação aos materiais de parede (Gráfico 7), pode-se observar que, entre as microcápsulas de óleos vegetais, $32 \%$ não especificaram o material de parede empregado, $21,5 \%$ de mistura de encapsulantes; $13 \%$ amido; $13 \%$ utilizaram como materiais de parede outros encapsulantes como gelatina, quitosana, alginato de sódio, beta-glucona, ciclodextrina, celulose; $11 \%$ empregavam goma arábica como material de parede; $9 \%$ maltodextrina; $2 \%$ caseinato de sódio ou caseína; $2 \%$ proteína do leite. Esses resultados são similares aos apresentados em estudo de Aghbashlo et al. (2013), em que os materiais de parede mais utilizados para encapsular óleos vegetais são as gomas, especialmente goma arábica ou acácia e maltodextrinas de diferentes equivalentes de dextroses. As duas patentes brasileiras que tratavam do encapsulamento de óleos não especificaram o tipo de material de parede empregado.

Gráfico 7 - Materiais de parede empregados no microencapsulamento nas patentes do Espacenet
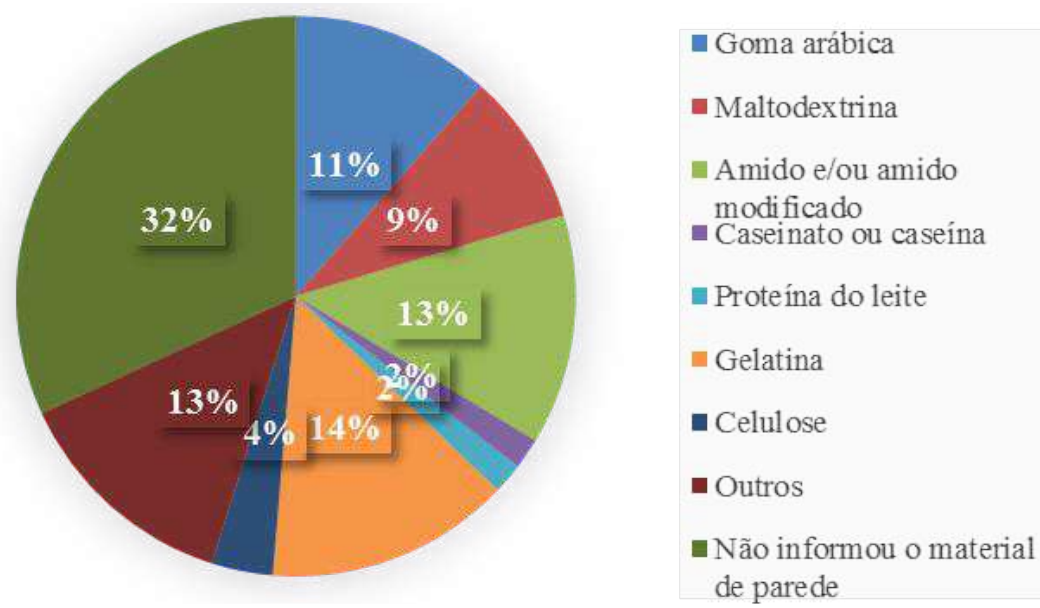

Fonte: Elaborado pelos autores deste artigo (2020)

\subsubsection{Origem da Tecnologia}

A porcentagem de patentes requeridas internacionalmente encontradas na base Espacenet (Gráfico 8A) revela que os documentos sobre essa tecnologia se concentram no Japão (32\%). Em segundo lugar está os Estados Unidos com 25\%, seguido da China com 22\%. No entanto, os EUA são os maiores produtores de óleos, com uma produção superior a 100 milhões de toneladas, e a China ocupa o terceiro lugar no ranking, com uma produção em torno de 60 milhões de toneladas (FAS, 2020a).

Vale ressaltar que o Brasil não está entre os detentores da tecnologia das 354 patentes analisadas nesta base de dados. Isso pode ser atribuído a duas razões: ou o Brasil não está preocupado em exportar sua tecnologia, ou ainda há uma incipiência da cultura de depósito de patentes. Essa estratégia brasileira pode ser perigosa, pois apenas o território brasileiro fica restrito para produção e comercialização, podendo a tecnologia desenvolvida pelo Brasil ser utilizada em qualquer outro país como domínio público. 
No entanto, em relação aos documentos depositados no INPI, o Brasil e a Alemanha ocupam o segundo lugar do ranking dos países detentores da tecnologia, com $20 \%$ de patentes depositadas (Gráfico 8B), perdendo apenas para os Estados Unidos (25\%). Essa informação está em consonância com o descrito pelo United States Department of Agriculture (FAS, 2020b), em que a produção brasileira de óleos é a segunda maior do mundo, com 77 milhões de toneladas. Neste estudo foi identificada a região de origem dos depositantes com o objetivo de avaliar quais regiões brasileiras são responsáveis por proteger a tecnologia de interesse (FAS, 2020a).

Apesar de a produção de óleos no país se concentrar na Região Norte (NETO, 2008; BRASIL, 2011; ALMEIDA, 2012), a Região Sudeste predomina em número de patentes depositadas no INPI, com três patentes do Estado de São Paulo. Esse resultado pode ser justificado pelo fato de a Região Sudeste ser a que mais investe no estudo dessa tecnologia. De acordo com Suave et al. (2006), há no Brasil 109 grupos de pesquisa em diferentes áreas do conhecimento que empregam técnicas de microencapsulamento. Entre eles, destacam-se grupos na Universidade de São Paulo (USP), da Universidade Estadual de Campinas (UNICAMP), da Universidade Federal de São Carlos (UFSCar), da Universidade Federal do Rio de Janeiro (UFRJ), da Universidade Federal do Rio Grande do Sul (UFRGS), da Universidade Federal do Rio Grande do Norte (UFRN), da Universidade Federal de Pernambuco (UFPE), da Universidade Federal de Goiás (UFG) e da Universidade Federal do Paraná (UFPR), além do Instituto de Pesquisas Tecnológicas (IPT) e da Empresa Brasileira de Pesquisa Agropecuária (EMBRAPA).

Gráfico 8 - Distribuição de depósitos de patentes por país de origem nas bases de dados do Espacenet $(\mathrm{A})$ e INPI $(\mathrm{B})$

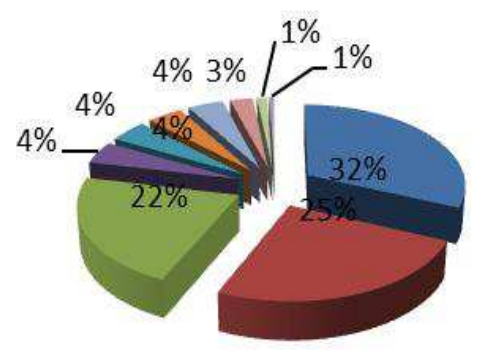

A

Japão
Estados Unidos
- China
- Alemanha
- Coréia do Sul
- Grã- Bretanha
- Canadá
- França
- Espanha
- Austrália

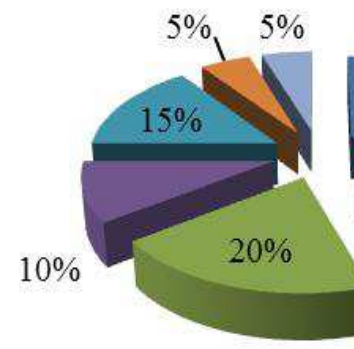

B
Estados Unidos

- Alemanha

- Brasil

Espanha

: Reino Unido

⿶ Japão

China

Fonte: Elaborado pelos autores deste artigo (2020)

\subsubsection{Detentores da Tecnologia de Produção}

No que tange aos detentores da tecnologia de produção, das 354 patentes internacionais, a empresa asiática Fuji Photo Film Co Ltd é a líder no ranking dos detentores da tecnologia de produção com 19 patentes, seguida da empresa Japonesa Canon KK, especializada em produtos de imagem e ópticos, com sete patentes (Gráfico 9A).

Em relação às patentes depositadas no banco nacional (Gráfico 9B), entre os 20 depositores, apenas quatro são de origem brasileira, e, entre estes, estão a Universidade de São Paulo com duas patentes e as empresas Dublauto Indústria e Comércio Ltda e Polymar Indústria Comércio Importação e Exportação Ltda. Pelo Gráfico 9B, nota-se que a Universidade de São Paulo, a empresa Papierfabrik August Koehler Ag, da Alemanha, e a Syngenta Limited, do Reino Unido, detêm o maior número de patentes no INPI. 
Gráfico 9 - Depósito de patentes relacionadas ao microencapsulamento de óleos, por detentores nas bases de dados do Espacenet (A) e INPI (B)

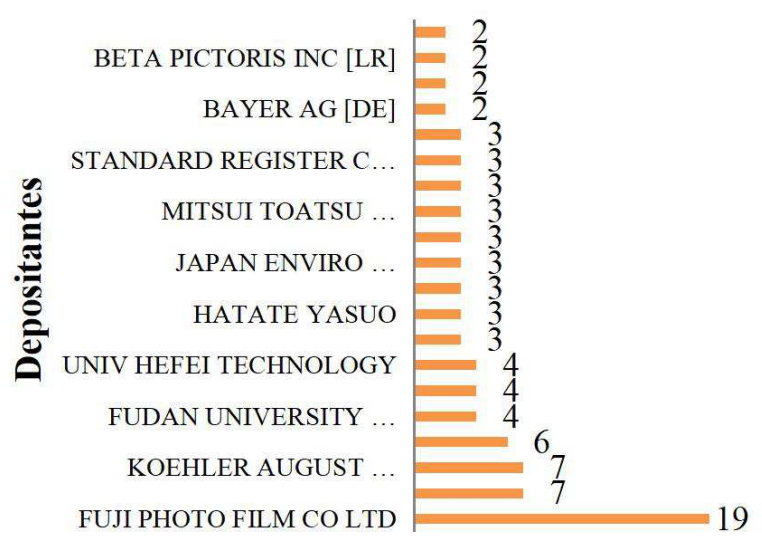

A

Número de patentes

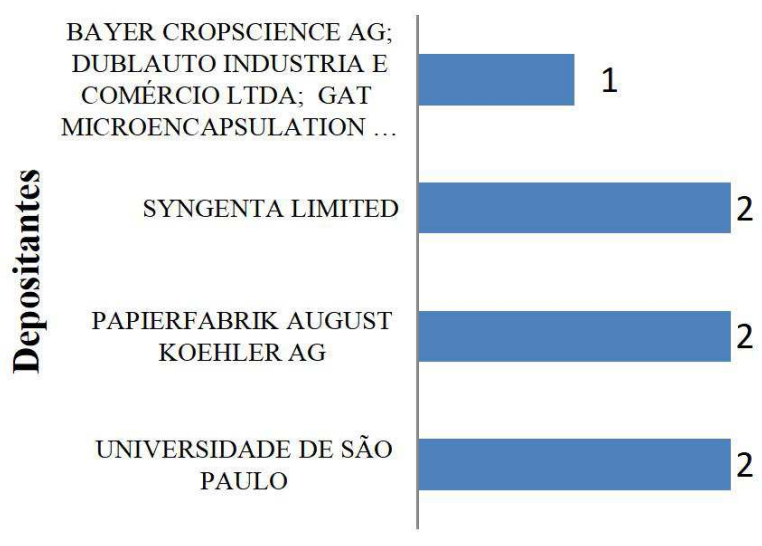

B
Número de patentes

Fonte: Elaborado pelos autores deste artigo (2020)

\subsubsection{Inventores da Tecnologia}

O Gráfico 10 relaciona o número de patentes por inventores de acordo com os dados disponíveis no Espacenet. O cenário é semelhante ao dos detentores de tecnologia, sendo possível observar que o principal inventor é também de origem asiática, com 10 patentes. O domínio de patentes, tanto por detentores como por inventores asiáticos, pode estar associado ao investimento maciço desses países, tanto na educação básica como na superior. A China, por exemplo, passou a desenvolver pesquisas dentro de suas próprias universidades e depois a comercializar os resultados. Na Coreia do Sul e em Taiwan, a importância dada à inovação é tão grande que os países passaram a produzir milhares de patentes por ano (MAHMOOD; SINGH, 2003).

Gráfico 10 - Inventores da tecnologia em relação ao microencapsulamento de óleos na base de dados do Espacenet

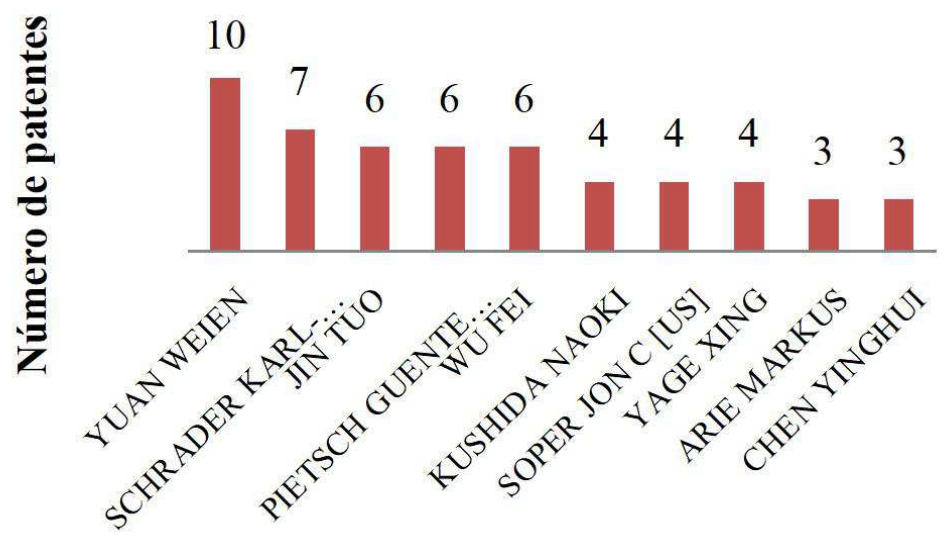

\section{Inventores}

Fonte: Elaborado pelos autores deste artigo (2020) 
Vale ressaltar que o atual crescimento econômico dos países asiáticos também pode atuar como fator de contribuição para o desenvolvimento de novas tecnologias e posteriores patentes. De acordo com a Organizações das Nações Unidas (ONU), as economias da Ásia e do Pacífico tiveram um crescimento moderado em 2013, após forte desaceleração em 2012, causada por fatores externos.

A busca de patentes na base de dados nacional revelou que os inventores Osvaldo de Freitas e Günter Pietsch detêm o maior número de patentes sobre a tecnologia em questão, apresentando duas patentes cada um. Vale ressaltar que nenhum dos dois é inventor independente, já que eles estão associados à Universidade de São Paulo, em São Paulo, e à empresa Papierfabrik August Koehler, na Alemanha. Os demais inventores do banco de dados nacional depositaram apenas uma patente cada um.

\section{Considerações Finais}

A partir da análise das patentes depositadas nos bancos de dados do Espacenet e do INPI, pode-se concluir que existe uma tendência ao crescimento de depósitos, indicando que muitas inovações têm sido realizadas, atestando, assim, a importância do microencapsulamento de óleos.

No que diz respeito à origem da tecnologia patenteada, verifica-se uma concentração nos países desenvolvidos, com domínio pelo Japão, Estados Unidos e China. Possivelmente, devido ao crescimento econômico e no setor de pesquisa e desenvolvimento, os países Asiáticos lideram entre as empresas e inventores com maior número de patentes. De acordo com as patentes depositadas no INPI, os países que mais depositaram no país foram EUA, Alemanha e Brasil. No âmbito nacional, este estudo prospectivo mostra que o número de patentes depositadas pelo Brasil é muito pequeno, revelando que o uso dessa tecnologia de microencapsulamento aparece como uma grande oportunidade de geração de patentes, principalmente pelas inúmeras possibilidades de aplicação dessa tecnologia.

O óleo de soja é o mais utilizado para o microencapsulamento de óleos no mundo, a área de aplicação mais empregada, tanto no mundo quanto no Brasil, é a indústria farmacêutica, spray-drying é a tecnologia de microencapsulamento predominante, e os materiais de parede mais empregados são gelatina, maltodextrina e amido.

\section{Referências}

AGHBASHLO, M. et al. Fish oil microencapsulation as in $\square$ uenced by spray dryer operational variables. International Journal of Food Science and Technology, [s.l.], v. 48, p. 1.707-1713, 2013.

AHN, J. et al. Optimization of microencapsulation of seed oil by response surface methodology. Food Chemistry, [s.l.], v. 107, p. 98-105, 2008.

ALMEIDA, J. R. Criação de valor sustentável e o óleo de palma no Brasil. 2012. 119 p. Dissertação (Mestrado) - Escola de Administração de Empresas de São Paulo, Fundação Getúlio Vargas, São Paulo, 2012. 
AZEREDO, H. M. C. Encapsulação: aplicação à tecnologia de alimentos. Alimentos e Nutrição, Araraquara, [s.l.], v. 16, p. 89-97, 2005.

BARRET, A. H. et al. Effect of various antioxidants, antioxidants levels, and encapsulation on the stability of fish and flaxseeds oil: assessment by fluorometric analysis. Journal of Food Processing and Preservation, [s.l.], v. 35, p. 349-358, 2001.

BRASIL. Ministério da Agricultura, Pecuária e Abastecimento - MAPA. Projeções do Agronegócio - 2010/2011 a 2020/2021. [2011].

BROCK, J. et al. Determinação experimental da viscosidade e condutividade térmica de óleos vegetais. Ciência e Tecnologia de Alimentos, [s.l.], v. 28, p. 564-570, 2008.

CALVO, P. et al. Microencapsulation of extra-virgin olive oil by spray-drying: In $\square$ uence of wall material and olive quality. European Journal of Lipid Science and Technology, [s.l.], v. 112, p. 852-858, 2010.

CARR, N. O.; HOOG, F. W. A manufacturer's perspective on selected palm-based products. Asia Pacific Journal Clinical Nutrition, [s.l.], v. 14, p. 381-386, 2005.

CARNEIRO, H. C. F. et al. Encapsulation efficiency and oxidative stability of flaxseed oil microencapsulated by spray drying using different combinations of wall materials. Journal of Food Engineering, [s.l.], v. 115, p. 443-451, 2013.

CASTRO, H. F. et al. Modificação de óleos e gorduras por biotransformação. Química Nova, [s.l.], v. 27, p. 146-156, 2004.

CHANG, C. et al. Release properties on gelatin-gum arabic microcapsules containing camphor oil with added polystyrene. Colloids and Surfaces B: Biointerfaces, [s.l.], v. 50, p. 136-140, 2006.

CONCEIÇÃO, M. M. et al. Rheological Behavior of Castor Oil Biodiesel. Eenergy \& Fuels, [s.l.], v. 19, 2005.

CRESCIMENTO sustentável de longo prazo para Ásia e Pacífico requer investimentos em políticas de proteção social. 2004. Disponível em: http://www.planalto.gov.br/ccivil_03/_ato2004-2006/2004/lei/ 110.973.htm. Acesso em: 18 mar. 2020.

DIAN, N. L. H. M.; SUDIN, N.; YUSOFF, M. S. A. Characteristics of Microencapsulated Palm-Based Oil as affected by type of wall material. Journal of Agriculture and Food Chemistry, [s.l.], v. 70, p. 422-426, 1996.

DOMIAN, E. et al. Influence of agglomeration on physical characteristics and oxidative stability of spray-dried oil powder with milk protein and trehalose wall material. Journal of Food Engineering, [s.l.], v. 125, p. 34-43, 2014.

DRUSCH, S.; MANNINO, S. Patent-based review on industrial approaches for the microencapsulation of oils rich in polyunsaturated fatty acids. Trends in Food Science \& Technology, [s.l.], v. 20, p. 237-244, 2009.

FARIA, E. A. Estudo da estabilidade térmica de óleos e gorduras vegetais por TG/DTG e DTA.

Eclética Química, [s.l.], v. 27, 2002.

FAS - FOREIGN AGRICULTURAL SERVICE. United States Department of Agriculture (USDA). Oilseeds and Products Annual: Brazil, 2019. (2020a). Disponível em: https://apps.fas.usda.gov/ psdonline/circulars/production.pdf. Acesso em: 18 mar. 2020. 
FAS - FOREIGN AGRICULTURAL SERVICE. United States Department of Agriculture (USDA). Oilseeds: World Markets and Trade, 2019. (2020b). Disponível em: https://apps.fas.usda.gov/ psdonline/circulars/oilseeds.pdf. Acesso em: 18 mar. 2020.

FERREIRA, C. D. et al. Itaciara Larroza Nunes . Morfologia de microcápsulas de óleo de palma bruto (Elaeis guineensis) obtidas através de microscopia eletrônica de varredura (MEV). In: XXIV CONGRESSO BRASILEIRO DE CIÊNCIA E TECNOLOGIA DE ALIMENTOS (XXIV CBCTA) e IV CONGRESSO DO INSTITUTO NACIONAL DE CIÊNCIA E TECNOLOGIA DE FRUTOS TROPICAIS, 2014, Aracaju. Anais [...]. Aracaju, 2014.

FERREIRA, C. D. et al. Physicochemical Characterization and Oxidative Stability of Microencapsulated Crude Palm Oil by Spray Drying. Food and Bioprocess Technology, [s.l.], v. 9, p. 124-136, 2016a.

FERREIRA, C. D. et al. Microcápsula de azeite de dendê. Patente: Privilégio de Inovação. Número do registro: BR1020160058888, título: "Microcápsula de azeite de dendê", Instituição de registro: INPI - Instituto Nacional da Propriedade Industrial. Depósito: 12/04/2016. Brasil. 2016b.

FERREIRA, C. D. et al. Método para obtenção de microcápsulas de azeite de dendê por spraydrying. Patente: Privilégio de Inovação. Número do registro: BR1020160085861, título: "Método para obtenção de microcápsulas de azeite de dendê por spray-drying", Instituição de registro: INPI Instituto Nacional da Propriedade Industrial. Depósito: 12/04/2016. Brasil. 2016c.

FROEHNER, S.; LEITHOLD, J.; LIMA JÚNIOR, L. F. Transesterificação de óleos vegetais: caracterização por cromatografia em camada delgada e densidade. Química Nova, [s.l.], v. 30, p. 2.016-2.019, 2007.

GALLARDO, G. et al. Microencapsulation of linseed oil by spray drying for functional food application. Food Research International, [s.l.], v. 52, p. 473-482, 2013.

GOUIN, S. Microencapsulation: industrial appraisal of existing technologies and trends. Trends in Food Science \& Technology, [s.l.], v. 15, p. 330-347, 2004.

HEINZELMANN, K.; FRANKE, K. Using freezing and drying techniques of emulsions for the microencapsulation of fish oil to improve oxidation stability. Colloids and Surfaces B: Biointerfaces, [s.l.], v. 12, p. 223-229, 1999.

JAFARI, S. M. et al. Nano-particle encapsulation of fish oil by spray drying. Food Research International, [s.l.], v. 41, p. 172-183, 2008.

JEYARANI, T.; YELLA REDDY, S. Preparation of Plastic Fats with Zero trans FA from Palm Oil. Journal of the American Oil Chemists' Society, [s.l.], v. 80, p. 1.107-1.113, 2003.

KAGAMI, Y. et al. Oxidative stability, structure, and physical characteristics of microcapsules formed by spray drying of fish oil with protein and dextrin wall materials. Journal of Food Science, [s.l.], v. 68, p. 2.248-2.255, 2003.

KEOGH, M. K. et al. Stability to Oxidation of Spray-Dried Fish Oil Powder Microencapsulated Using Milk Ingredients. Journal of Food Science, [s.l.], v. 66, p. 217-224, 2001.

KOEHLER, A. PAPIERFABRIK: Método de microencapsulamento de óleos hidrófobos com partes reativas de corantes de sistemas de reação de corantes dissolvidas nos mesmos e microcápsula. Alemanha, PI 8801228-0 A2, 1988. 
MAHMOOD, P. I.; SINGH J. Technological Dynamism in Asia. Research Policy, [s.l.], v. 32, v. 1.031-1.054, 2003.

MENDONÇA C. G.; RAETANO, C. G.; MENDONÇA, C. G. Tensão superficial estática de soluções aquosas com óleos minerais e vegetais utilizados na agricultura. Revista Engenharia Agrícola, [s.l.], v. 27, p. 16-23, 2007.

MOREIRA, G. E. G. Obtenção e caracterização de extrato microencapsulado de resíduo agroindustrial de acerola. 2007. 72 f. Dissertação (Mestrado em Engenharia Química) Universidade Federal do Rio Grande do Norte, Natal, 2007.

MÜLLER, P. S. Microencapsulação de óleo essencial de laranja. 2011. 98 f. Dissertação (Mestrado em Tecnologia de Alimentos) - Universidade Federal do Paraná, Curitiba, PR, 2011.

NETO, M. Matérias-primas para biocombustíveis, 2008. Disponível em: http://materiaprimas. blogspot.com/2008/07/leo-de-palma.html. Acesso em: 18 mar. 2020.

OLIVEIRA, G. R. et al. Prospecção tecnológica: processo de liofilização na indústria de alimentos. Revista Geintec, [s.l.], v. 3, p. 92-102, 2012.

OSBORNE, A. P. GB Pat. 1090973, 1964.

PARIZE, A. L. Desenvolvimento de sistemas microparticulares e de filmes a base de quitosina e corante natural cúrcuma. 2009. 187 f. Tese (Doutorado em Química) - Universidade Federal de Santa Catarina, Florianópolis, 2009.

POLAVARAPU, S. et al. Physicochemical characterization and oxidative stability of fish oil and fish oil-extra virgin olive oil microencapsulated by sugar beet pectin. Food Chemistry, [s.l.], v. 127, p. 1.694-1.705, 2011.

QUINTELLA, C. M. et al. Cadeia do biodiesel da bancada à indústria: uma visão geral com prospecção de tarefas e oportunidades para P\&D\&I. Química Nova, [s.l.], v. 32, p. 793-808, 2009.

QUISPE-CONDORI, S.; SALDAÑA, M. D. A.; TEMELLI, F. Microencapsulation of flax oil with zein using spray and freeze drying. LWT - Food Science and Technology, [s.l.], v. 44, p. 1.880-1.887, 2011.

RABELLO, F. F. P. Microencapsulação de ingredientes alimentícios. Revista Agrogeoambiental, [s.l.], 2009.

SHEN, Z. et al. Oxidative stability of microencapsulated fish oil powders stabilized by blends of chitosan, modified starch, and g. lucose. Journal of Agriculture and Food Chemistry, [s.l.], v. 58, p. 4.487-4.493, 2010.

SUAVE, J. et al. Microencapsulação: Inovação em diferentes áreas. Revista Saúde e Ambiente, [s.l.], v. 7, p. 12-20, 2006.

TONON, R. V.; GROSSO, C. R. F.; HUBINGER, M. D. Influence of emulsion composition and inlet air temperature on the microencapsulation of flaxseed oil by spray drying. Food Research International, [s.l.], v. 44, p. 282-289, 2011.

WANG, R.; TIAN, Z.; CHEN, L. A novel process for microencapsulation of fish oil with barley protein. Food Research International, [s.l.], v. 44, p. 2.735-2.741, 2011. 


\section{Sobre os Autores}

\section{Camila Duarte Ferreira Ribeiro}

E-mail: camiladuartef@ufba.br

Doutora em Alimentos, Nutrição e Saúde pela Universidade Federal da Bahia em 2018.

Endereço profissional: Escola de Nutrição da UFBA, Rua Basílio da Gama, s/n, Canela. Salvador, BA. CEP: 40110-040.

\section{Luciane Santos Sousa}

E-mail: lusousa.bromato@gmail.com; lusousa.bromato@gmail.com

Mestre em Ciência de Alimentos pela Universidade Federal da Bahia em 2016.

Endereço profissional: Faculdade de farmácia, UFBA, Avenida Ademar de Barros, Laboratório de Pescados e Cromatografia Aplicada, sala 109, Ondina, Salvador, BA. CEP: 41820-785.

\section{Ísis Maria Pereira Borges}

E-mail: isismariaborges@hotmail.com

Mestre em Ciência de Alimentos pela Universidade Federal da Bahia em 2015.

Endereço profissional: UniFTC, Avenida Luís Viana Filho, n. 8.812, Paralela, Salvador, BA. CEP: 41741-590.

\section{Itaciara Larroza Nunes}

E-mail: itaciara.nunes@ufsc.br

Doutora em Ciência de Alimentos pela Universidade Estadual de Campinas em 2005.

Endereço profissional: Universidade Federal de Santa Catarina, Centro de Ciências Agrárias. Rodovia Admar Gonzaga, n. 1.346, Itacorubi, Florianópolis, SC. CEP: 88034-000. 